\title{
A NOTE ON POSITIVE SOLUTIONS FOR CONSERVATION LAWS WITH SINGULAR SOURCE
}

\author{
D. AMADORI AND G. M. COCLITE \\ (Communicated by Walter Craig)
}

Abstract. We consider the Cauchy problem for the scalar conservation law

$$
\partial_{t} u+\partial_{x} f(u)=\frac{1}{g(u)}, \quad t>0, x \in \mathbb{R},
$$

with $g \in C^{1}(\mathbb{R}), g(0)=0, g(u)>0$ for $u>0$, and assume that the initial datum $u_{0}$ is nonnegative.

We show the existence of entropy solutions that are positive a.e. by means of an approximation of the equation that preserves positive solutions and by passing to the limit using a monotonicity argument. The difficulty lies in handling the singularity of the right-hand side (the source term) as $u$ possibly vanishes at the initial time. The source term is shown to be locally integrable.

Moreover, we prove a uniqueness and stability result for the above equation.

\section{INTRODUCTION}

We consider the initial value problem for the hyperbolic conservation law with singular source

$$
\begin{cases}\partial_{t} u+\partial_{x} f(u)=\frac{1}{g(u)}, & t>0, x \in \mathbb{R}, \\ u(0, x)=u_{0}(x), & x \in \mathbb{R},\end{cases}
$$

where we assume that

$$
\begin{array}{ll}
f \in C^{1}(\mathbb{R}), & \\
g \in C^{1}(\mathbb{R}), & g(0)=0, \quad \xi>0 \Longrightarrow g(\xi)>0, \quad \int_{0}^{\infty} g(\xi) d \xi=\infty, \\
& u_{0} \geq 0, \quad u_{0} \in L^{\infty}(\mathbb{R}) .
\end{array}
$$

In several traffic flow [2] and gasdynamic models [8], formulated in terms of hyperbolic conservation laws $3,5,6$, the unknown $u(t, x)$ represents a density and when the vacuum is experienced one has $u(t, x)=0$. In (1.1) the source term $1 / g(u)$ blows up in the presence of a vacuum. The singular problem (1.1) is a first step toward a better understanding of the isentropic gasdynamics equations in Eulerian coordinates. The next steps clearly consist of the extensions of the present result to systems and to changing sign initial conditions.

Received by the editors August 26, 2011.

2010 Mathematics Subject Classification. Primary 35B25, 35B09, 35L65.

Key words and phrases. Singular nonlinear problems, positive solutions, conservation laws. 
Our result is strictly related to the existence one in [4. Indeed there the authors prove the existence of nonnegative solutions for the integrodifferential problem

$$
\begin{cases}\partial_{t} u+\partial_{x} f(u)=\int_{0}^{1} K(x, y) \frac{1}{g(u(t, y))} d y, & t>0, x \in(0,1), \\ u(t, 0)=u(t, 1)=0, & t>0, \\ u(0, x)=u_{0}(x), & x \in(0,1),\end{cases}
$$

where $K$ is a nonnegative integral kernel (say the Green's function of a differential operator). Formally, if

$$
K \longrightarrow \delta_{\{x=y\}}
$$

we get (1.1).

Let us finally mention the following two papers. Schonbek 10] considered a singularity depending on $x$ and not on $u$. Natalini, Sinestrari, and Tesei [9] studied the blowup in the case of locally bounded sources.

Here the main difficulty is to prove that the source term $1 / g(u)$ lies in $L_{l o c}^{1}$, even if the initial condition is identically 0 (see Lemma 3.1). This is necessary in order to give a meaning to (1.1) in the distributional sense.

Let us give the definition of an entropy solution for (1.1).

Definition 1.1. A function $u:[0, \infty) \times \mathbb{R} \rightarrow \mathbb{R}$ is an entropy solution of (1.1) if

i) $u \in L^{\infty}((0, T) \times \mathbb{R})$ for every $T>0$;

ii) $u \neq 0$ a.e., and $1 / g(u) \in L_{\text {loc }}^{1}((0, \infty) \times \mathbb{R})$;

iii) for every convex entropy $\eta \in C^{2}(\mathbb{R})$ with flux $q$ defined by $q^{\prime}=\eta^{\prime} f^{\prime}$, the inequality

$$
\int_{0}^{\infty} \int_{\mathbb{R}}\left(\eta(u) \partial_{t} \varphi+q(u) \partial_{x} \varphi+\frac{\eta^{\prime}(u)}{g(u)} \varphi\right) d t d x+\int_{\mathbb{R}} \eta\left(u_{0}(x)\right) \varphi(0, x) d x \geq 0
$$

holds for every nonnegative test function $\varphi \in C_{c}^{\infty}\left(\mathbb{R}^{2}\right)$.

In the rest of the paper we will frequently use the function $G$ defined as follows:

$$
G(\xi)=\int_{0}^{\xi} g(s) d s, \quad \xi \in[0, \infty) .
$$

In particular, we observe that

$$
G(0)=G^{\prime}(0)=0, \quad \xi \neq 0 \Longrightarrow G^{\prime}(\xi)>0, \quad \lim _{\xi \rightarrow \infty} G(\xi)=\infty ;
$$

hence $G$ is increasing and invertible in $[0, \infty)$ and its inverse $G^{-1}$ is defined in $[0, \infty)$.

Let us state our main result.

Theorem 1.1. Let (1.2) hold. Then the initial value problem (1.1) has an entropy solution $u$, in the sense of Definition 1.1, such that

$$
0 \leq G^{-1}(t) \leq u(t, x) \leq G^{-1}\left(t+G\left(\left\|u_{0}\right\|_{L^{\infty}(\mathbb{R})}\right)\right), \quad \text { for a.e. } t \geq 0 \text { and } x \in \mathbb{R} .
$$

Moreover, let $T>0$ and assume that

$$
\sup \left\{-\frac{g^{\prime}(w)}{g^{2}(w)}: w \in(0, k]\right\}<\infty, \quad \text { for all } k>0 .
$$


If $u$ and $v$ are entropy solutions of (1.1) obtained in correspondence of the initial conditions $u_{0}, v_{0} \in L^{\infty}(\mathbb{R}), u_{0}, v_{0} \geq 0$, then, for any $R>0$ and $0 \leq t \leq T$, the Kružkov estimate holds:

$$
\int_{-R}^{R}|u(t, x)-v(t, x)| d x \leq e^{\gamma_{T} t} \int_{-R-L_{T} t}^{R+L_{T} t}\left|u_{0}-v_{0}\right| d x
$$

where

$$
\begin{aligned}
\gamma_{T} & :=\sup \left\{-\frac{g^{\prime}(w)}{g^{2}(w)}: w \in\left(0, \mathcal{G}_{T}\right]\right\}, \\
L_{T} & :=\max \left\{\left|f^{\prime}(w)\right|: w \in\left[0, \mathcal{G}_{T}\right]\right\}, \\
\mathcal{G}_{T} & :=G^{-1}\left(T+G\left(\max \left\{\left\|u_{0}\right\|_{L^{\infty}(\mathbb{R})},\left\|v_{0}\right\|_{L^{\infty}(\mathbb{R})}\right\}\right)\right) .
\end{aligned}
$$

We prove our existence result studying the solutions of the following approximation of (1.1):

$$
\begin{cases}\partial_{t} u_{\varepsilon}+\partial_{x} f\left(u_{\varepsilon}\right)=\frac{1}{g\left(u_{\varepsilon}\right)+\varepsilon}, & t>0, x \in \mathbb{R}, \\ u_{\varepsilon}(0, x)=u_{0}(x), & x \in \mathbb{R} .\end{cases}
$$

The existence of a unique bounded nonnegative entropy solution $u_{\varepsilon}$ has been proved in [7.

Our argument is based on the precise knowledge of the properties of the entropy solution to (1.7); see [7, 1]. One interesting feature of the approximated problem is the monotonicity of the family $\left\{u_{\varepsilon}\right\}_{\varepsilon>0}$ with respect to $\varepsilon$ that gives the convergence of all of the family to the entropy weak solution of (1.1) and not just the convergence of a subsequence. The main point of our analysis is the convergence of the approximated source terms $1 /\left(g\left(u_{\varepsilon}\right)+\varepsilon\right)$ due to the unboundedness of $1 / g(u)$.

The assumption (1.5) on the source term $g$ is trivially satisfied if $g^{\prime}(u) \geq 0$, for instance with $g(u)=u$. An example of a source term that satisfies (1.2) and not (1.5) is given by $1 / g(u)$ with

$$
g(u)= \begin{cases}u^{3}\left(2+\sin \left(\frac{1}{u}\right)\right), & \text { if } u \neq 0 \\ 0, & \text { if } u=0\end{cases}
$$

Notice also that the uniqueness does not hold in the class of solutions with no given sign. As a simple example, consider $g(u)=u, u(0, \cdot) \equiv 0$, and $u(t, x)=u(t)=$ $\pm \sqrt{2 t}$.

The note is organized as follows. Section 2 is dedicated to some a priori estimates uniform with respect to $\varepsilon$ on the solutions of the approximated problem (1.7). In Section 3 we prove our main result.

\section{A PRIORI ESTIMATES}

This section is devoted to some estimates on $u_{\varepsilon}$ uniform with respect to $\varepsilon$. Let us begin by introducing the notation

$$
G_{\varepsilon}(\xi)=G(\xi)+\varepsilon \xi, \quad \xi \in \mathbb{R}, \varepsilon>0 .
$$

Thanks to (1.3), we know that

$$
G_{\varepsilon}(0)=0, \quad G_{\varepsilon}^{\prime}=g+\varepsilon, \quad \xi \geq 0 \Longrightarrow G_{\varepsilon}^{\prime}(\xi), G_{\varepsilon}(\xi) \geq 0 .
$$


In particular $G_{\varepsilon}$ is increasing and invertible in $[0, \infty)$ and its inverse $G_{\varepsilon}^{-1}$ is defined in $[0, \infty)$.

Lemma 2.1 ( $L^{\infty}$-estimate). We have that

$$
0 \leq G_{\varepsilon}^{-1}(t) \leq u_{\varepsilon}(t, x) \leq G^{-1}\left(t+G\left(\left\|u_{0}\right\|_{L^{\infty}(\mathbb{R})}\right)\right)=: M(t)
$$

for every $\varepsilon>0, t \geq 0$, and $x \in \mathbb{R}$.

Proof. We have only to verify that the functions

$$
v(t, x)=M(t), \quad w(t, x)=G_{\varepsilon}^{-1}(t), \quad t \geq 0, x \in \mathbb{R},
$$

are a super and a subsolution of (1.7). We observe that

$$
\begin{array}{r}
\partial_{t} v+\partial_{x} f(v)-\frac{1}{g(v)+\varepsilon}=v^{\prime}-\frac{1}{g(v)+\varepsilon} \geq v^{\prime}-\frac{1}{g(v)}=0, \\
\partial_{t} w+\partial_{x} f(w)-\frac{1}{g(w)+\varepsilon}=w^{\prime}-\frac{1}{g(w)+\varepsilon}=0,
\end{array}
$$

and obviously at time $t=0$,

$$
w(0, \cdot)=0 \leq u_{0} \leq\left\|u_{0}\right\|_{L^{\infty}(\mathbb{R})}=v(0, \cdot) .
$$

Therefore the claim follows from the comparison principle for scalar conservation laws with bounded sources [7].

Lemma 2.2 (Monotonicity). The family $\left\{u_{\varepsilon}\right\}_{\varepsilon>0}$ is nondecreasing as $\varepsilon \rightarrow 0$; namely, the following inequality holds:

$$
u_{\varepsilon} \leq u_{\varepsilon^{\prime}}, \quad \text { a.e. in }[0, \infty) \times \mathbb{R}
$$

for every $\varepsilon \geq \varepsilon^{\prime}>0$.

Proof. Let $\varepsilon \geq \varepsilon^{\prime}>0$. We have only to observe that

$$
\frac{1}{g(\cdot)+\varepsilon} \leq \frac{1}{g(\cdot)+\varepsilon^{\prime}}
$$

The claim follows from the comparison principle for scalar conservation laws with bounded sources [7]; see also Proposition 1.2.2 in [9].

Lemma 2.3 ( $L_{l o c}^{1}$-estimate on the source). Let $a<b$ and $T>0$. The following inequality holds for every $0<\varepsilon \leq 1$ :

$$
\left\|\frac{1}{\varepsilon+g\left(u_{\varepsilon}\right)}\right\|_{L^{1}((0, T) \times(a, b))} \leq K_{T} C_{T, a, b},
$$

where

$$
K_{T}:=M(T+1)+\sup _{[0, M(T+1)]}|f|,
$$

$M(T)$ is defined in (2.2) and $C_{T, a, b}$ is a positive constant depending only on $T, a$, and $b$.

Proof. Let $\varphi=\varphi(t, x)$ be a test function such that

$$
\begin{aligned}
& \varphi \in C^{\infty}\left(\mathbb{R}^{2}\right), \quad 0 \leq \varphi \leq 1, \\
& \varphi(t, x)= \begin{cases}1, & \text { if }(t, x) \in[0, T] \times[a, b], \\
0, & \text { if }(t, x) \in \mathbb{R}^{2} \backslash((-1, T+1) \times(a-1, b+1)) .\end{cases}
\end{aligned}
$$


Thanks to (2.2), since $u_{\varepsilon}$ is a distributional solution of (1.7), we have

$$
\begin{aligned}
& \left\|\frac{1}{\varepsilon+g\left(u_{\varepsilon}\right)}\right\|_{L^{1}((0, T) \times(a, b))}=\int_{0}^{T} \int_{a}^{b} \frac{d t d x}{\varepsilon+g\left(u_{\varepsilon}\right)} \leq \int_{0}^{\infty} \int_{\mathbb{R}} \frac{\varphi(t, x)}{\varepsilon+g\left(u_{\varepsilon}\right)} d t d x \\
& =-\int_{0}^{\infty} \int_{\mathbb{R}} \partial_{t} \varphi(t, x) u_{\varepsilon} d t d x-\int_{0}^{\infty} \int_{\mathbb{R}} \partial_{x} \varphi(t, x) f\left(u_{\varepsilon}\right) d t d x \underbrace{-\int_{\mathbb{R}} \varphi(0, x) u_{0}(x) d x}_{\leq 0} \\
& \leq\left\|u_{\varepsilon}\right\|_{L^{\infty}((0, T+1) \times \mathbb{R})}\left\|\partial_{t} \varphi\right\|_{L^{1}\left(\mathbb{R}^{2}\right)}+\left\|f\left(u_{\varepsilon}\right)\right\|_{L^{\infty}((0, T+1) \times \mathbb{R})}\left\|\partial_{x} \varphi\right\|_{L^{1}\left(\mathbb{R}^{2}\right)} \\
& \leq\left\{M(T+1)+\sup _{[0, M(T+1)]}|f|\right\}\|\varphi\|_{W^{1,1}\left(\mathbb{R}^{2}\right)} \leq K_{T}\|\varphi\|_{W^{1,1}\left(\mathbb{R}^{2}\right)} .
\end{aligned}
$$

By taking the infimum over all possible test functions $\varphi$ that satisfy (2.6), we end up with (2.4).

\section{Proof of Theorem 1.1}

Let us begin with the convergence of the families $\left\{u_{\varepsilon}\right\}_{\varepsilon>0}$ and $\left\{1 /\left(g\left(u_{\varepsilon}\right)+\varepsilon\right)\right\}_{\varepsilon>0}$.

Lemma 3.1. There exists a function $u \in L_{l o c}^{\infty}((0, \infty) \times \mathbb{R})$ such that

$$
u_{\varepsilon} \longrightarrow u \quad \text { a.e. and in } L_{l o c}^{p}((0, \infty) \times \mathbb{R}), 1 \leq p<\infty \text { as } \varepsilon \rightarrow 0 \text {. }
$$

Moreover, $u$ is nonnegative a.e. in $[0, \infty) \times \mathbb{R}$ and satisfies (1.4).

In addition, we have that

$$
\begin{aligned}
& u \neq 0 \quad \text { a.e. in }[0, \infty) \times \mathbb{R}, \\
& \frac{1}{g(u)} \in L_{l o c}^{1}((0, \infty) \times \mathbb{R}), \\
& \left\|\frac{1}{g(u)}\right\|_{L^{1}((0, T) \times(a, b))} \leq K_{T} C_{T, a, b}, \\
& \frac{1}{\varepsilon+g\left(u_{\varepsilon}\right)} \longrightarrow \frac{1}{g(u)} \text { in } L_{l o c}^{1}((0, \infty) \times \mathbb{R}),
\end{aligned}
$$

for every $T>0$ and $a<b$, where $K_{T}$ is defined in (2.5) and $C_{T, a, b}$ is a positive constant depending only on $T, a$, and $b$.

Proof. Let us introduce the function

$$
u=\sup _{\varepsilon>0} u_{\varepsilon} .
$$

Due to Lemma 2.1. (1.4) holds. Moreover, thanks to Lemma 2.2.

$$
u_{\varepsilon} \longrightarrow u \quad \text { a.e. in }[0, \infty) \times \mathbb{R} \text {. }
$$

Therefore, (1.4) and the Dominated Convergence Theorem together give (3.1).

Now let $T>0, a<b$. We begin by proving (3.2), (3.3), and (3.4). Consider the function

$$
\widetilde{g}:[0, \infty) \times \mathbb{R} \rightarrow \mathbb{R}_{+} \cup\{+\infty\}, \quad \widetilde{g}(t, x)= \begin{cases}\frac{1}{g(u(t, x))}, & \text { if } u(t, x) \neq 0 \\ \infty, & \text { if } u(t, x)=0\end{cases}
$$


Clearly, from (3.1) one has

$$
\widetilde{g} \geq 0, \quad \frac{1}{\varepsilon+g\left(u_{\varepsilon}\right)} \longrightarrow \widetilde{g} \text { a.e. in }[0, \infty) \times \mathbb{R} .
$$

Therefore Lemma 2.3 and Fatou's Lemma give

$$
\int_{0}^{T} \int_{a}^{b} \widetilde{g} d t d x \leq \liminf _{k} \int_{0}^{T} \int_{a}^{b} \frac{1}{\varepsilon+g\left(u_{\varepsilon}\right)} d t d x \leq K_{T} C_{T, a, b},
$$

which means

$$
\widetilde{g} \in L_{l o c}^{1}((0, \infty) \times \mathbb{R}) .
$$

As a consequence we have that $\widetilde{g}$ is finite a.e. and therefore (3.2), (3.3), and (3.4) hold, with $\widetilde{g}=\frac{1}{g(u)}$.

We conclude by proving (3.5). Let $\psi: \mathbb{R} \rightarrow \mathbb{R}$ be a cutoff function such that

$$
\psi \in C^{\infty}(\mathbb{R}), \quad \psi^{\prime} \leq 0 \leq \psi \leq 1, \quad \psi(u)= \begin{cases}1, & \text { if } 0 \leq u \leq \frac{1}{2}, \\ 0, & \text { if } u \geq 1,\end{cases}
$$

and let $\sigma>0$ be a parameter to be chosen later. We observe that

$$
\begin{aligned}
& \left\|\frac{1}{\varepsilon+g\left(u_{\varepsilon}\right)}-\frac{1}{g(u)}\right\|_{L^{1}((0, T) \times(a, b))} \\
& \leq\left\|\psi\left(\frac{u_{\varepsilon}}{\sigma}\right) \frac{1}{\varepsilon+g\left(u_{\varepsilon}\right)}\right\|_{L^{1}((0, T) \times(a, b))}+\left\|\psi\left(\frac{u}{\sigma}\right) \frac{1}{g(u)}\right\|_{L^{1}((0, T) \times(a, b))} \\
& \quad+\left\|\left(\psi\left(\frac{u_{\varepsilon}}{\sigma}\right)-\psi\left(\frac{u}{\sigma}\right)\right) \frac{1}{g(u)}\right\|_{L^{1}((0, T) \times(a, b))} \\
& \quad+\left\|\left(1-\psi\left(\frac{u_{\varepsilon}}{\sigma}\right)\right)\left(\frac{1}{\varepsilon+g\left(u_{\varepsilon}\right)}-\frac{1}{g(u)}\right)\right\|_{L^{1}((0, T) \times(a, b))} .
\end{aligned}
$$

Since

$$
\begin{aligned}
\left|\left(\psi\left(\frac{u_{\varepsilon}}{\sigma}\right)-\psi\left(\frac{u}{\sigma}\right)\right) \frac{1}{g(u)}\right| & \leq \frac{2}{g(u)}, \\
\left|\left(1-\psi\left(\frac{u_{\varepsilon}}{\sigma}\right)\right)\left(\frac{1}{\varepsilon+g\left(u_{\varepsilon}\right)}-\frac{1}{g(u)}\right)\right| & \leq \frac{1}{g(u)}+\max _{w \in\left[\frac{\sigma}{2}, M(T)\right]} \frac{1}{g(w)},
\end{aligned}
$$

and the functions on the right-hand side are summable in $(0, T) \times(a, b)$ (see (3.3)), the Dominated Convergence Theorem and (3.1) give

$$
\begin{aligned}
\limsup _{\varepsilon \rightarrow 0+}\left\|\left(\psi\left(\frac{u_{\varepsilon}}{\sigma}\right)-\psi\left(\frac{u}{\sigma}\right)\right) \frac{1}{g(u)}\right\|_{L^{1}((0, T) \times(a, b))} & =0, \\
\limsup _{\varepsilon \rightarrow 0+}\left\|\left(1-\psi\left(\frac{u_{\varepsilon}}{\sigma}\right)\right)\left(\frac{1}{\varepsilon+g\left(u_{\varepsilon}\right)}-\frac{1}{g(u)}\right)\right\|_{L^{1}((0, T) \times(a, b))} & =0 .
\end{aligned}
$$

We claim that$$
\left\|\psi\left(\frac{u_{\varepsilon}}{\sigma}\right) \frac{1}{\varepsilon+g\left(u_{\varepsilon}\right)}\right\|_{L^{1}((0, T) \times(a, b))} \leq C_{T, a, b} \int_{0}^{M(T+1)} \psi\left(\frac{s}{\sigma}\right)\left[1+\left|f^{\prime}(s)\right|\right] d s,
$$$$
\left\|\psi\left(\frac{u}{\sigma}\right) \frac{1}{g(u)}\right\|_{L^{1}((0, T) \times(a, b))} \leq C_{T, a, b} \int_{0}^{M(T+1)} \psi\left(\frac{s}{\sigma}\right)\left[1+\left|f^{\prime}(s)\right|\right] d s,
$$

for some constant $C_{T, a, b}>0$ depending only on $T, a$, and $b$. 
To prove (3.10), let $\varphi=\varphi(t, x)$ be a test function satisfying (2.6). Since the function

$$
u \longmapsto-\int_{0}^{u} \psi\left(\frac{s}{\sigma}\right) d s
$$

is convex in $[0, \infty)$ and $u_{\varepsilon}$ is nonnegative, from the definition of an entropy solution we have

$$
\begin{aligned}
& \left\|\psi\left(\frac{u_{\varepsilon}}{\sigma}\right) \frac{1}{\varepsilon+g\left(u_{\varepsilon}\right)}\right\|_{L^{1}((0, T) \times(a, b))} \\
& =\int_{0}^{T} \int_{a}^{b} \psi\left(\frac{u_{\varepsilon}}{\sigma}\right) \frac{1}{\varepsilon+g\left(u_{\varepsilon}\right)} d t d x \\
& \leq \int_{0}^{\infty} \int_{\mathbb{R}} \psi\left(\frac{u_{\varepsilon}}{\sigma}\right) \frac{\varphi(x)}{\varepsilon+g\left(u_{\varepsilon}\right)} d t d x \\
& \leq-\int_{0}^{\infty} \int_{\mathbb{R}} \partial_{t} \varphi(t, x) \int_{0}^{u_{\varepsilon}(t, x)} \psi\left(\frac{s}{\sigma}\right) d s d t d x \underbrace{-\int_{\mathbb{R}} \varphi(0, x) \int_{0}^{u_{0}(x)} \psi\left(\frac{s}{\sigma}\right) d s d x}_{\leq 0} \\
& -\int_{0}^{\infty} \int_{\mathbb{R}} \partial_{x} \varphi(t, x) \int_{0}^{u_{\varepsilon}(t, x)} \psi\left(\frac{s}{\sigma}\right) f^{\prime}(s) d s d t d x \\
& \leq\left\|\partial_{t} \varphi\right\|_{L^{1}\left(\mathbb{R}^{2}\right)} \int_{0}^{M(T+1)} \psi\left(\frac{s}{\sigma}\right) d s+\left\|\partial_{x} \varphi\right\|_{L^{1}\left(\mathbb{R}^{2}\right)} \int_{0}^{M(T+1)} \psi\left(\frac{s}{\sigma}\right)\left|f^{\prime}(s)\right| d s \\
& \leq\|\varphi\|_{W^{1,1}\left(\mathbb{R}^{2}\right)} \int_{0}^{M(T+1)} \psi\left(\frac{s}{\sigma}\right)\left[1+\left|f^{\prime}(s)\right|\right] d s .
\end{aligned}
$$

Therefore (3.10) is proved.

Now let us prove (3.11). Fatou's Lemma and (3.10) together give

$$
\begin{aligned}
\left\|\psi\left(\frac{u}{\sigma}\right) \frac{1}{g(u)}\right\|_{L^{1}((0, T) \times(a, b))} & =\left\|\liminf _{\varepsilon \rightarrow 0+} \psi\left(\frac{u_{\varepsilon}}{\sigma}\right) \frac{1}{\varepsilon+g\left(u_{\varepsilon}\right)}\right\|_{L^{1}((0, T) \times(a, b))} \\
& \leq \liminf _{\varepsilon \rightarrow 0+}\left\|\psi\left(\frac{u_{\varepsilon}}{\sigma}\right) \frac{1}{\varepsilon+g\left(u_{\varepsilon}\right)}\right\|_{L^{1}((0, T) \times(a, b))} \\
& \leq C_{T, a, b} \int_{0}^{M(T)} \psi\left(\frac{s}{\sigma}\right)\left[1+\left|f^{\prime}(s)\right|\right] d s,
\end{aligned}
$$

which is (3.11).

At last, using (3.8), (3.9), (3.10), and (3.11) in (3.7),

$$
\begin{aligned}
& \limsup _{\varepsilon \rightarrow 0+}\left\|\frac{1}{\varepsilon+g\left(u_{\varepsilon}\right)}-\frac{1}{g(u)}\right\|_{L^{1}((0, T) \times(a, b))} \\
& \quad \leq \limsup _{\varepsilon \rightarrow 0+}\left\|\psi\left(\frac{u_{\varepsilon}}{\sigma}\right) \frac{1}{\varepsilon+g\left(u_{\varepsilon}\right)}\right\|_{L^{1}((0, T) \times(a, b))}+\left\|\psi\left(\frac{u}{\sigma}\right) \frac{1}{g(u)}\right\|_{L^{1}((0, T) \times(a, b))} \\
& \quad \leq 2 C_{T, a, b} \int_{0}^{M(T+1)} \psi\left(\frac{s}{\sigma}\right)\left[1+\left|f^{\prime}(s)\right|\right] d s .
\end{aligned}
$$

Since this estimate holds for every $\sigma>0$, as $\sigma \rightarrow 0$ we get (3.5).

Proof of Theorem 1.1. We begin by proving that the function $u$ introduced in Lemma 3.1 is an entropy solution of (1.1) in the sense of Definition 1.1. Due to Lemmas 2.1 and 3.1. we have only to verify the entropy inequalities. 
Let $\varphi \in C_{c}^{\infty}\left(\mathbb{R}^{2}\right), \varphi \geq 0$, and $\eta \in C^{2}(\mathbb{R})$ be a convex entropy with flux $q$ defined by $q^{\prime}=\eta^{\prime} f^{\prime}$. The following inequality holds:

$$
\int_{0}^{\infty} \int_{\mathbb{R}}\left(\eta\left(u_{\varepsilon}\right) \partial_{t} \varphi+q\left(u_{\varepsilon}\right) \partial_{x} \varphi+\frac{\eta^{\prime}\left(u_{\varepsilon}\right)}{g\left(u_{\varepsilon}\right)+\varepsilon} \varphi\right) d t d x+\int_{\mathbb{R}} \eta\left(u_{0}(x)\right) \varphi(0, x) d x \geq 0 .
$$

Thanks to the Dominated Convergence Theorem and Lemma 3.1, as $\varepsilon \rightarrow 0$ we have that

$$
\int_{0}^{\infty} \int_{\mathbb{R}}\left(\eta(u) \partial_{t} \varphi+q(u) \partial_{x} \varphi+\frac{\eta^{\prime}(u)}{g(u)} \varphi\right) d t d x+\int_{\mathbb{R}} \eta\left(u_{0}(x)\right) \varphi(0, x) d x \geq 0
$$

therefore $u$ is an entropy solution of (1.1) in the sense of Definition 1.1.

Notice that, if $u$ is an entropy solution of (1.1), then the following inequality

$$
\partial_{t}|u-k|+\partial_{x}(\operatorname{sign}(u-k)(f(u)-f(k)))-\operatorname{sign}(u-k) \frac{1}{g(u)} \leq 0
$$

holds in the sense of distributions for every $k \in \mathbb{R}$. Indeed, let $k \in \mathbb{R}$ and $\left\{\eta_{n}\right\}_{n \in \mathbb{N}} \subset$ $C^{2}(\mathbb{R})$ be a sequence of convex entropies such that

$$
\begin{aligned}
& \eta_{n} \rightarrow|\cdot-k| \quad \text { uniformly on every compact subset of } \mathbb{R}, \\
& \eta_{n}^{\prime} \rightarrow \operatorname{sign}(\cdot-k), \quad \text { in } L_{l o c}^{1}(\mathbb{R}), \\
& \left\|\eta_{n}^{\prime}\right\|_{L^{\infty}(\mathbb{R})} \leq 1, \quad n \in \mathbb{N} .
\end{aligned}
$$

Introducing the notation

$$
q_{n}(\xi)=\int_{k}^{\xi} \eta_{n}^{\prime}(s) f^{\prime}(s) d s, \quad \xi \in \mathbb{R}
$$

we have also

$$
q_{n} \rightarrow \operatorname{sign}(\cdot-k)(f(\cdot)-f(k)), \quad \text { uniformly on every compact subset of } \mathbb{R} .
$$

Let $\phi \in C^{1}((0, \infty) \times \mathbb{R})$ be a nonnegative test function with compact support. Since $u$ is an entropy solution of (1.1) we have

$$
\int_{0}^{\infty} \int_{\mathbb{R}}\left(\eta_{n}(u) \partial_{t} \phi+q_{n}(u) \partial_{x} \phi+\frac{\eta_{n}^{\prime}(u)}{g(u)} \phi\right) d t d x \geq 0 .
$$

As $n \rightarrow \infty$, using the boundedness of $u$, the summability of $1 / g(u)$, and the Dominate Convergence Theorem we have that

$$
\int_{0}^{\infty} \int_{\mathbb{R}}\left(|u-k| \partial_{t} \phi+\operatorname{sign}(u-k)(f(u)-f(k)) \partial_{x} \phi+\frac{\operatorname{sign}(u-k)}{g(u)} \phi\right) d t d x \geq 0,
$$

which is (3.14).

We conclude by proving the uniqueness and stability of positive entropy solutions of (1.1) under the assumption (1.5). Let $R>0,0 \leq t \leq T$, $u$ and $v$ be two entropy solutions of (1.1) obtained in correspondence of the initial conditions $u_{0}, v_{0} \in L^{\infty}(\mathbb{R}), u_{0}, v_{0} \geq 0$ respectively. For every $\varepsilon>0$, let $u_{\varepsilon}, v_{\varepsilon}$ be the two entropy solutions of (1.7) obtained in correspondence of the initial conditions $u_{0}, v_{0}$ respectively. From the stability results of [7] we know that

$$
\int_{-R}^{R}\left|u_{\varepsilon}(t, x)-v_{\varepsilon}(t, x)\right| d x \leq e^{\gamma_{\varepsilon, T} t} \int_{-R-L_{T} t}^{R+L_{T} t}\left|u_{0}-v_{0}\right| d x
$$


where

$$
\gamma_{\varepsilon, T}:=\sup \left\{-\frac{g^{\prime}(w)}{(g(w)+\varepsilon)^{2}}: w \in\left(0, \mathcal{G}_{T}\right]\right\} .
$$

If $g^{\prime}(w)<0$ for some $w \in\left(0, \mathcal{G}_{T}\right]$, one simply has

$$
0<\gamma_{\varepsilon, T} \leq \gamma_{T}, \quad \varepsilon>0
$$

hence from (3.15) we get

$$
\int_{-R}^{R}\left|u_{\varepsilon}(t, x)-v_{\varepsilon}(t, x)\right| d x \leq e^{\gamma_{T} t} \int_{-R-L_{T} t}^{R+L_{T} t}\left|u_{0}-v_{0}\right| d x
$$

and (1.6) follows from (3.1).

On the other hand, if $g^{\prime}(w) \geq 0$ for all $w \in\left(0, \mathcal{G}_{T}\right]$, one has that $\gamma_{\varepsilon, T} \leq 0$ and $\gamma_{T} \leq 0$. Therefore, we can affirm that (1.6) holds with Lipschitz constant 1 in place of $e^{\gamma_{T} t}$, which is sufficient to prove the uniqueness of the solution.

In order to prove estimate (1.6), with the possibly negative decay $e^{\gamma_{T} t}$, one can use a direct approach in the spirit of [7, Theorem 1]; see also [5, 1]. We cannot deduce it from (3.15) since it may happen that $\liminf _{\varepsilon \rightarrow 0} \gamma_{\varepsilon, T}>\gamma_{T}$. This is the case for $g(u)=u^{2}$.

Here a difficulty lies in the fact that the source term is not bounded but only locally integrable, and this occurs in the dependence of the source on the state variable $u$.

Let $\phi \geq 0$ be $C^{1}$ with compact support in $(0, T) \times \mathbb{R}$. The proof is mainly devoted to deriving the inequality

$$
\begin{aligned}
\int_{0}^{T} \int_{\mathbb{R}} & \left\{|u(t, x)-v(t, x)| \partial_{t} \phi(t, x)\right. \\
& +\operatorname{sign}(u(t, x)-v(t, x)) \cdot[f(u(t, x))-f(v(t, x))] \partial_{x} \phi(t, x) \\
\quad & \left.+\operatorname{sign}(u(t, x)-v(t, x)) \cdot\left[\frac{1}{g(u(t, x))}-\frac{1}{g(v(t, x))}\right] \phi(t, x)\right\} d x d t \geq 0 .
\end{aligned}
$$

Let $N$ be a constant such that the support of $\phi$ is contained in $\Omega:=(0, T) \times(-N, N)$. Define the $C^{1}$ function $\varphi$ as

$$
\varphi(t, x ; \tau, y)=\phi\left(\frac{t+\tau}{2}, \frac{x+y}{2}\right) \lambda_{h}\left(\frac{t-\tau}{2}, \frac{x-y}{2}\right)
$$

where

$$
\lambda_{h}(a, b)=\delta_{h}(a) \delta_{h}(b), \quad \delta_{h}(a)=\frac{1}{h} \delta\left(\frac{a}{h}\right),
$$

and $\delta: \mathbb{R} \rightarrow \mathbb{R}$ is a function such that

$$
0 \leq \delta \leq 1, \quad \delta \in C^{\infty}(\mathbb{R}), \quad \int_{\mathbb{R}} \delta(x) d x=1, \quad x \notin[-1,1] \Longrightarrow \delta(x)=0 .
$$

Observe that if $h$ is sufficiently small, the support of $\varphi$ is contained in the open set $\mathcal{G}=\Omega \times \Omega$. The analogue of the inequality (3.4) in [7] is given by

$$
\sum_{i=1}^{4} \int_{\mathcal{G}} F_{i}(t, x ; \tau, y ; u(t, x), v(\tau, y)) \lambda_{h}\left(\frac{t-\tau}{2}, \frac{x-y}{2}\right) d x d t d y d \tau \geq 0
$$


with $F_{3} \equiv 0$ and

$$
\begin{aligned}
& F_{1}(t, x ; \tau, y ; u, v)=|u-v| \partial_{t} \phi\left(\frac{t+\tau}{2}, \frac{x+y}{2}\right) \\
& F_{2}(t, x ; \tau, y ; u, v)=\operatorname{sign}(u-v)[f(u)-f(v)] \partial_{x} \phi\left(\frac{t+\tau}{2}, \frac{x+y}{2}\right), \\
& F_{4}(t, x ; \tau, y ; u, v)=\operatorname{sign}(u-v)\left[\frac{1}{g(u)}-\frac{1}{g(v)}\right] \phi\left(\frac{t+\tau}{2}, \frac{x+y}{2}\right) .
\end{aligned}
$$

Now, if $|u|,\left|v_{1}\right|,\left|v_{2}\right| \leq M(T)$, then there exists a constant $C>0$ depending on $f, \phi, M(T)$ such that for $i=1,2$ we have

$$
\begin{aligned}
\left|F_{i}\left(t, x ; \tau, y ; u, v_{1}\right)-F_{i}\left(t, x ; t, x ; u, v_{2}\right)\right| & \leq C\left[\left(|t-\tau|+|x-y|+\left|v_{1}-v_{2}\right|\right)\right] \\
& =: F\left(t, x ; \tau, y ; v_{1}, v_{2}\right) .
\end{aligned}
$$

On this term, following [7, p. 225] one has

$$
\lim _{h \rightarrow 0} \int_{\mathcal{G}} F(t, x ; \tau, y ; v(\tau, y), v(t, x)) \lambda_{h}\left(\frac{t-\tau}{2}, \frac{x-y}{2}\right) d t d x d \tau d y=0
$$

and then one obtains for $i=1,2$ :

$$
\begin{aligned}
\lim _{h \rightarrow 0} \int_{\mathcal{G}} F_{i}(t, x ; \tau, y ; u(t, x), v & (\tau, y)) \lambda_{h}\left(\frac{t-\tau}{2}, \frac{x-y}{2}\right) d t d x d \tau d y \\
& =4 \int_{\Omega} F_{i}(t, x ; t, x ; u(t, x), v(t, x)) d t d x .
\end{aligned}
$$

On the term with $i=4$, we cannot use the same argument because $1 / g(u)$ is not Lipschitz continuous for $u>0$. Let us set, for $u>0$ and $v>0$ :

$$
k(u, v)= \begin{cases}\operatorname{sign}(u-v)\left[\frac{1}{g(u)}-\frac{1}{g(v)}\right], & \text { if } u \neq v, \\ 0, & \text { if } u=v,\end{cases}
$$

and write

$$
\begin{aligned}
F_{4}(t, x ; \tau, y ; u(t, x), v(\tau, y))= & k(u(t, x), v(\tau, y)) \phi\left(\frac{t+\tau}{2}, \frac{x+y}{2}\right) \\
= & k(u(t, x), v(t, x)) \phi(t, x) \\
& +k(u(t, x), v(\tau, y))\left[\phi\left(\frac{t+\tau}{2}, \frac{x+y}{2}\right)-\phi(t, x)\right] \\
& +[k(u(t, x), v(\tau, y))-k(u(t, x), v(t, x))] \phi(t, x) .
\end{aligned}
$$

Let us denote by $\mathcal{I}_{4}$ the term with $i=4$ in (3.17) and write it as a sum

$$
\mathcal{I}_{4}=\mathcal{J}_{1}+\mathcal{J}_{2}+\mathcal{J}_{3}
$$

related to the terms (3.18), (3.19), and (3.20), respectively. The first term turns out to be independent of $h$ :

$$
\mathcal{J}_{1}=4 \int_{\Omega} k(u(t, x), v(t, x)) \phi(t, x) d t d x .
$$

Now we show that $\mathcal{J}_{2}(h)$ and $\mathcal{J}_{3}(h)$ vanish as $h \rightarrow 0$. The function in (3.19) is estimated by

$$
\left(\frac{1}{g(u(t, x))}+\frac{1}{g(v(\tau, y))}\right)(|x-y|+|t-\tau|) .
$$


Let us evaluate the corresponding integrals. With the change of variables

$$
(s, \xi, \sigma, \eta)=\left(t, x, \frac{x-y}{2}, \frac{t-\tau}{2}\right),
$$

we deduce that

$$
\begin{aligned}
\int_{\mathcal{G}} & \frac{1}{g(u(t, x))}(|x-y|+|t-\tau|) \lambda_{h}\left(\frac{t-\tau}{2}, \frac{x-y}{2}\right) d t d x d \tau d y \\
& =\left(\int_{\Omega} \frac{1}{g(u(s, \xi))} d s d \xi\right) \cdot\left(\int_{-h}^{h} \int_{-h}^{h} 8(|\eta|+|\sigma|) \lambda_{h}(\sigma, \eta) d \eta d \sigma\right) ;
\end{aligned}
$$

the first integral is finite because of the summability of $1 / g(u)$, while the second vanishes as $h \rightarrow 0$. The same analysis shows that, as $h \rightarrow 0$,

$$
\int_{\mathcal{G}} \frac{1}{g(v(\tau, y))}(|x-y|+|t-\tau|) \lambda_{h}\left(\frac{t-\tau}{2}, \frac{x-y}{2}\right) d t d x d \tau d y \longrightarrow 0
$$

so that also $\mathcal{J}_{2}(h) \rightarrow 0$. Finally, using again (3.21), we have

$$
\mathcal{J}_{3}=4 \int_{-h}^{h} \int_{-h}^{h} \lambda_{h}(\sigma, \eta) \psi(\sigma, \eta) d \sigma d \eta
$$

where

$$
\psi(\sigma, \eta)=\int_{\Omega}[k(u(t, x), v(x-2 \eta, t-2 \sigma))-k(u(t, x), v(t, x))] \phi(t, x) d t d x .
$$

We claim that $\psi(\sigma, \eta) \rightarrow 0$ as $(\sigma, \eta) \rightarrow(0,0)$. This clearly implies that $\mathcal{J}_{3}(h) \rightarrow 0$.

To prove that $\psi(\sigma, \eta) \rightarrow 0$, it is enough to prove that for any sequence $\left(\sigma_{j}, \eta_{j}\right) \rightarrow$ $(0,0)$ there exists a subsequence, still denoted by $\left(\sigma_{j}, \eta_{j}\right)$, such that $\psi\left(\sigma_{j}, \eta_{j}\right) \rightarrow 0$.

Step 1. Recall that $v \in L^{\infty}(\Omega)$ and $\Omega$ is bounded. We use the continuity of translation in $L_{l o c}^{1}$ to deduce that, for any sequence $\left(\sigma_{j}, \eta_{j}\right) \rightarrow(0,0)$, there exists a subsequence, still denoted by $\left(\sigma_{j}, \eta_{j}\right)$, such that

$$
v\left(s-2 \sigma_{j}, \xi-2 \eta_{j}\right) \rightarrow v(s, \xi) \quad \text { for a.e. }(s, \xi) .
$$

By the continuity of $k$, the integrand

$$
G_{j}(s, \xi):=\left[k\left(u(s, \xi), v\left(s-2 \sigma_{j}, \xi-2 \eta_{j}\right)\right)-k(u(s, \xi), v(s, \xi))\right] \phi(s, \xi)
$$

vanishes a.e. as $j \rightarrow \infty$.

Step 2. Let $\varepsilon>0$. By the absolute continuity of the integral, there exists a $\delta>0$ such that if $E \subset \Omega$ is measurable and $|E|<\delta$, then

$$
\int_{E} \frac{1}{g(u(s, \xi))} d s d \xi<\varepsilon, \quad \int_{E} \frac{1}{g(v(s, \xi))} d s d \xi<\varepsilon .
$$

Notice that

$$
\int_{E}\left|G_{j}(s, \xi)\right| d s d \xi \leq C \int_{E} \frac{2}{g(u(s, \xi))}+\frac{1}{g(v(s, \xi))} d s d \xi+C \int_{E_{j}} \frac{1}{g(v(s, \xi))} d s d \xi,
$$

where $C=\|\phi\|_{L^{\infty}((0, \infty) \times \mathbb{R})}$ and $E_{j}=E-2\left(\sigma_{j}, \eta_{j}\right)$. Clearly $\left|E_{j}\right|=|E|<\delta$. Then

$$
\int_{E}\left|G_{j}(s, \xi)\right| d s d \xi \leq 4 C \varepsilon, \quad \forall j .
$$


Step 3. Let $\delta>0$ be given as in Step 2. We apply the Severini-Egoroff Theorem to the sequence $G_{j}$ and deduce that there exists $E_{o} \subset \Omega$, measurable with $\left|E_{o}\right|<\delta$, such that

$$
G_{j}(s, \xi) \rightarrow 0 \quad \text { uniformly on } \Omega \backslash E_{o}
$$

Therefore

$$
\left|\psi\left(\sigma_{j}, \eta_{j}\right)\right| \leq \int_{E_{o}}\left|G_{j}(s, \xi)\right| d s d \xi+\int_{\Omega \backslash E_{o}}\left|G_{j}(s, \xi)\right| d s d \xi,
$$

where the last integral vanishes as $j \rightarrow \infty$, thanks to the uniform convergence.

Using also (3.22), we can conclude that there exists $j_{\varepsilon}$ such that, for all $j \geq j_{\varepsilon}$, one has

$$
\left|\psi\left(\sigma_{j}, \eta_{j}\right)\right| \leq 4 C \varepsilon+\varepsilon
$$

This concludes the proof of (3.16). To complete the stability proof, we use (1.5) together with (3.16) and get

$$
\begin{aligned}
\int_{0}^{T} \int_{\mathbb{R}}\{ & |u(t, x)-v(t, x)| \partial_{t} \phi(t, x) \\
& +\operatorname{sign}(u(t, x)-v(t, x))[f(u(t, x))-f(v(t, x))] \partial_{x} \phi(t, x) \\
& \left.+\gamma_{T}|u(t, x)-v(t, x)| \phi(t, x)\right\} d x d t \geq 0 .
\end{aligned}
$$

Following [7, p. 228], a suitable choice of the test function $\phi$ leads to a Gronwalltype estimate for the quantity

$$
s \mapsto \int_{-R-L_{T}(t-s)}^{R+L_{T}(t-s)}|u(s, x)-v(s, x)| d x
$$

and finally to (1.6).

\section{REFERENCES}

[1] D. Amadori, L. Gosse, and G. Guerra. Godunov-type approximation for a general resonant balance law with large data. J. Differential Equations 198 (2) (2004), 233-274. MR2038581 (2004m:65114)

[2] A. Aw and M. Rascle. Resurrection of "second order" models of traffic flow. SIAM J. Appl. Math. 60 (3) (2000), 916-938. MR.1750085(2001a:35111)

[3] A. Bressan. Hyperbolic Systems of Conservation Laws. The one-dimensional Cauchy problem. Oxford Lecture Series in Mathematics and its Applications 20. Oxford University Press, Oxford, 2000. MR1816648(2002d:35002)

[4] G. M. Coclite and M. M. Coclite. Conservation laws with singular nonlocal sources. J. Differential Equations 250 (10) (2011), 3831-3858. MR2774070(2012b:35190)

[5] C. M. Dafermos. Hyperbolic conservation laws in continuum physics. Grundlehren der Mathematischen Wissenschaften 325. Springer-Verlag, Berlin, 2010. MR2574377 (2011i:35150)

[6] H. Holden and N. H. Risebro. Front tracking for hyperbolic conservation laws. Applied Mathematical Sciences 152. Springer-Verlag, New York, 2002. MR1912206 (2003e:35001)

[7] S. N. Kružkov. First order quasilinear equations with several independent variables. Mat. Sb. (N.S.) 81 (123) (1970), 228-255. MR0267257(42:2159)

[8] T. P. Liu and J. A. Smoller. On the vacuum state for the isentropic gas dynamics equations. Adv. in Appl. Math. 1(4) (1980), 345-359. MR603135 (83a:35065) 
[9] R. Natalini, C. Sinestrari, and A. Tesei. Incomplete blowup of solutions of quasilinear hyperbolic balance laws. Arch. Rational Mech. Anal. 135 (3) (1996), 259-296. MR1418466 (98d:35135)

[10] M. E. Schonbek. Existence of solutions to singular conservation laws. SIAM J. Math. Anal. 15 (6) (1984), 1125-1139. MR762969 (86c:35099)

Department of Pure \& Applied Mathematics, University of L'Aquila, Via Vetoio 1, 67010 Coppito (L'Aquila), Italy

E-mail address: amadori@univaq.it

$U R L$ : www.univaq.it/ amadori/

Department of Mathematics, University of Bari, Via E. Orabona 4, 70125 Bari, Italy

E-mail address: coclitegm@dm.uniba.it

$U R L$ : www.dm.uniba.it/Members/coclitegm/ 